\title{
Wide Range Control of Microstructure and Mechanical Properties of Carbon Nanotube Forests: A Comparison Between Fixed and Floating Catalyst CVD Techniques
}

\author{
Onnik Yaglioglu,* Anyuan Cao, A. John Hart, Rod Martens, and A. H. Slocum
}

Vertically aligned carbon nanotube (CNT) forests may be used as miniature springs, compliant thermal interfaces, and shock absorbers, and for these and other applications it is vital to understand how to engineer their mechanical properties. Herein is investigated how the diameter and packing density within CNT forests govern their deformation behavior, structural stiffness, and elastic energy absorption properties. The mechanical behavior of low-density CNT forests grown by fixed catalyst CVD methods and highdensity CNT forests grown by a floating catalyst CVD method are studied by in situ SEM compression testing and tribometer measurements of forcedisplacement relationships. Low-density and small-diameter CNT columns (fixed catalyst) exhibit large plastic deformation and can be pre-deformed to act as springs within a specified elastic range, whereas high-density and large-diameter CNT columns (floating catalyst) exhibit significant elastic recovery after deformation. In this work the energy absorption capacity of CNT forests is tuned over three orders of magnitude and it is shown that CNT forest density can be tuned over a range of conventional foam materials, but corresponding stiffness is $\sim 10 \times$ higher. It is proposed that the elastic behavior of CNT forests is analogous to open-cell foams and a simple model is presented. It is also shown that this model can be useful as a firstorder design tool to establish design guidelines for the mechanical properties of CNT forests and selection of the appropriate synthesis method.

\section{Introduction}

The mechanical properties and deformation mechanisms of vertically aligned CNTs (VA-CNT), also named CNT forests or CNT turfs, have recently attracted widespread attention. A thorough understanding of the mechanical properties is important for potential applications such as energy absorption, ${ }^{[1-3]}$ electromechanical probing,,$^{[4,5]}$ compliant contact structures for semiconductor packaging ${ }^{[6]}$ and compression-modulated filter membranes. ${ }^{[7]}$ Among the various CNT growth techniques including arc discharge and laser ablation, ${ }^{[8,9]}$ chemical vapor deposition (CVD) technique is the most suitable method for scalable industrial applications where a large number of CNT assemblies having micro to macro lengths is desirable. CVD techniques under the right conditions yield vertically aligned CNTs without external alignment efforts such as an applied electric field during growth. When the catalyst density is high, CNTs self-organize due to crowding at the start of growth to form a vertically-aligned forest. ${ }^{[5]}$

The CVD growth technique for CNT forests can be divided into two main approaches, namely fixed catalyst CVD and floating catalyst CVD. In the fixed catalyst CVD technique, a thin film of catalyst material, typically a transition metal, is deposited onto a support layer such as $\mathrm{Al}_{2} \mathrm{O}_{3} \cdot{ }^{[10-14]}$ Both layers can be successively deposited by evaporation or sputtering onto a flat substrate (e.g., a silicon wafer). The carbon source is typically a hydrocarbon gas (e.g., acetylene or ethylene) carried into a CVD furnace along with an inert gas at 700-800 C. In the floating catalyst CVD technique, a metallorganic catalyst precursor (e.g., ferrocene) is introduced in the gas phase instead of being deposited onto the growth substrate prior to the CVD process. ${ }^{[15-24]}$ The carbon source (e.g., xylene) is typically evaporated from the same solution containing the catalyst, and the mixed vapor is carried with an inert gas onto the growth substrate, which is typically a silicon wafer covered by an oxide film. In both cases, the CVD furnace temperature is typically 600-900 C.

Recent studies imply that the elastic behavior of CNT forests grown by CVD can be dramatically different and that 
there is no universal deformation mechanism. For example, forests grown by fixed catalyst methods typically result in irreversible plastic deformation upon compression; ${ }^{[25-29]}$ whereas forests grown by floating catalyst methods have large recoverable deformation, ${ }^{[1,3,7,30-33]}$ and are resistant to fatique which makes this technique attractive for applications where repeated loading is critical. ${ }^{[32]}$ In both cases CNT forests typically exhibit the formation of wave-like buckles, which accumulate with increasing applied force. This was first reported by Cao et al. ${ }^{[1]}$ for floating catalyst forests, and then reported in many papers including the references mentioned above. The first known insitu SEM observation of this buckle formation was reported in a Ph.D. thesis by Yaglioglu ${ }^{[5]}$ where the periodic and circumferential formation of the buckles was observed. Similar observations were later made by Hutchens et al. ${ }^{[29]}$ Stiffness tuning of VA-CNT structures grown with fixed catalyst recipe was first demonstrated by Yaglioglu, ${ }^{[5]}$ upon which this paper is based, utilizing the fact that introduction of hydrogen into the furnace during certain periods of growth has a significant effect on catalyst activity. ${ }^{[34,35]}$ Recently, stiffness tuning was also demonstrated for a floating catalyst system by modifying hydrogen flow rate. ${ }^{[33]}$

Importantly, there still lacks a unifying investigation of how the elastic properties of CNT forests can be related quantitatively to the diameter and packing density of the CNTs, and how important parameters such as energy absorption can be tuned by these parameters. Also, each study on the mechanical properties of CNT forest so far investigated only one type of growth recipe and there has been no reports comparing different growth recipes. The present study is therefore motivated by the need to determine the limits to mechanical properties of CNT forests that are established by both fixed and floating catalyst recipes. This is done using lithographically patterned CNT micropillars ("columns") as a model system to investigate how mechanical properties depend on the diameter and packing density of the CNTs. These key structural parameters are adjusted by the annealing conditions that precede the CVD growth step, and the design of the growth process (i.e., fixed vs. floating). We use in situ SEM imaging during micro-compression testing to compare the deformation modes of CNT forests grown by different recipes, as well as relate the elastic properties of the CNT forests to quantitative measures of the CNT size and density. We show that tuning the CNT diameter and density results in profound differences in both deformation behavior and elastic properties. We demonstrate that the elastic moduli of CNT forests can be tuned across two orders of magnitude, and the energy absorption capacity can range over 3 orders of magnitude. We also show that the elastic behavior of CNT forests can be modeled like an open-cell foam, and that a design space for CNT forests can be established based on the CNT diameter and density, with reasonable agreement to experimental results despite the complex mechanics that govern the collective deformation of large numbers of CNTs.

\section{Results and Discussion}

We studied VA-CNT forest columns grown by three different methods, which resulted in substantially different CNT diameter and packing density. The three methods are: 1) the fixed catalyst CVD method where growth substrates were heated in flowing argon, and hydrogen was introduced shortly before $\mathrm{C}_{2} \mathrm{H}_{4}$ was added as the carbon source; 2) the fixed catalyst CVD method where growth substrates were heated in flowing helium and hydrogen, and $\mathrm{C}_{2} \mathrm{H}_{4}$ was later added as the carbon source; and 3) the floating catalyst CVD method where the growth substrate was heated in flowing argon, and a mixture of ferrecone/xylene was supplied during the growth period. In each procedure, the height of the CNT columns is determined by the duration of the growth step when the carbon source is flowing. More details about the growth processses can be found in the experimental section. Throughout this paper, the first variation of the fixed catalyst process is referred to as "argon anneal", and the second variation is referred to as "hydrogen anneal".

The mechanical properties of the CNT forests grown by each technique were characterized by compressing CNT columns of various cross-sectional areas. A mask was designed with an array of different sized features such that geometric effects on mechanical properties could be tested on the same substrate, thereby eliminating run-to-run variation. The CNT columns were tested both in-situ SEM to visualize the deformation behavior, and ex-situ with a tribometer, which enabled accurate force and displacement measurents.

The three CVD methods mentioned above result in CNT forests with different morphologies, i.e. different CNT diameter and packing density. These are very important attributes, and as discussed in this paper, they have strong implications on the deformation mechanism and enable wide-range tuning of the mechanical properties of CNT forests. A SEM image of CNT columns grown by the floating catalyst method is shown in Figure 1a, and close up SEM images of the CNTs resulting from each technique are shown in Figure 1b. The corresponding average CNT diameter and average CNT-CNT spacing measurements are listed in Table 1. We found that the fixed catalyst method with the hydrogen anneal results in $\sim 20 \mathrm{X}$ higher areal density of CNTs compared to the fixed catalyst method with the argon anneal, but with $\sim 1 / 3^{\text {rd }}$ of the average CNT diameter. This translates into $\sim 2 \mathrm{X}$ structural density when comparing the two fixed catalyst methods. This difference is attributed to the interaction of $\mathrm{H}_{2}$ with the catalyst during the annealing step, where exposure to $\mathrm{H}_{2}$ before $\mathrm{C}_{2} \mathrm{H}_{4}$ influences the dewetting of the Fe film into nanoparticles and possibly promotes a higher catalyst activity. On the other hand, the floating catalyst CVD method yields a much higher structural density compared to both growth recipes employing fixed catalyst CVD methods, namely 25X compared to argon anneal and $13.3 \mathrm{X}$ compared to hydrogen anneal method. This is because the floating catalyst results in much larger CNT diameters and larger number of CNT walls. Thus, we state that the fixed catalyst method yields low density CNT forests, and the floating catalyst method yields high density CNT forests. These represent the measured limits to the mechanical properties of CNT forests as discussed in the following sections.

\subsection{Deformation Mechanism of Low-Density CNT Columns Grown by the Fixed Catalyst CVD Method}

To gain insight into the deformation mechanism, the columns were first compressed inside the SEM and the deformation was 


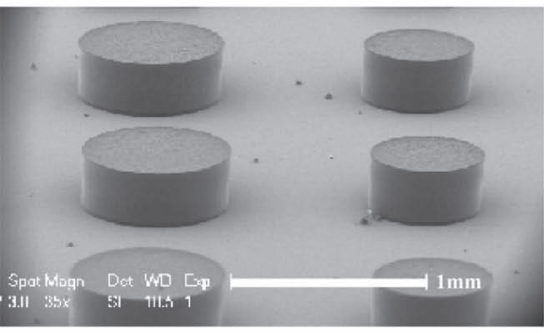

(a)
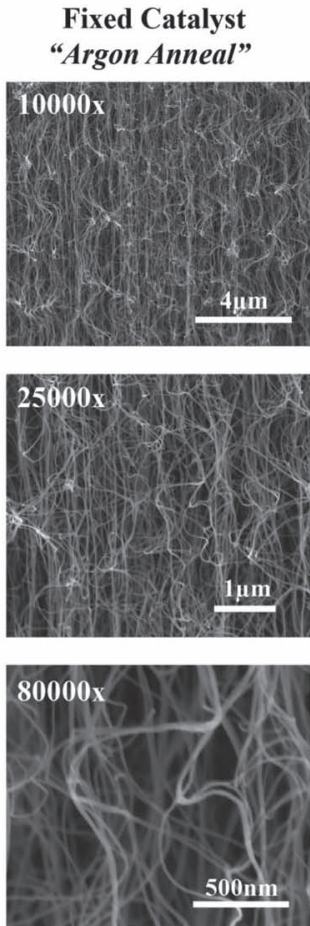

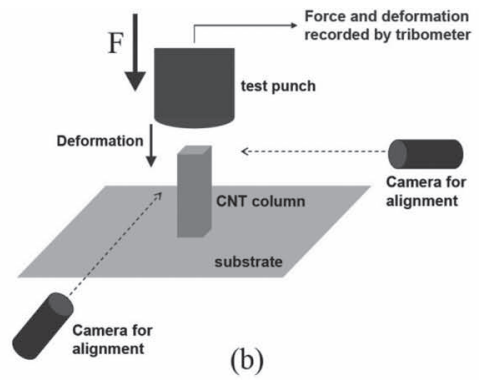

Floating Catalyst "Hydrogen Anneal"
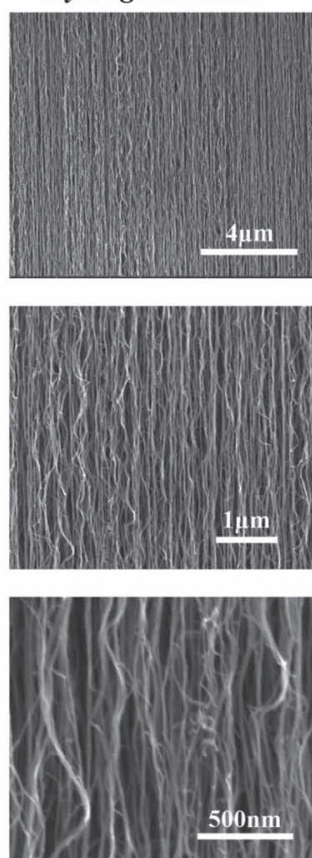

(c)
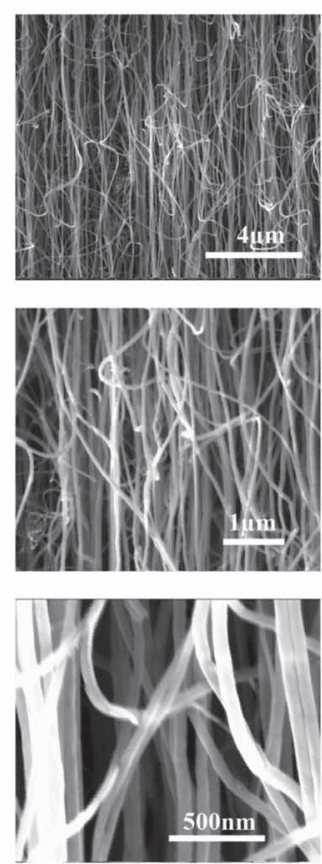

Figure 1. a) SEM image of CNT columns grown with the floating catalyst method. b) Test setup to characterize the mechanical properties of CNT columns. In the case of the in-situ SEM tests, alignment of the test probe to the CNT column is accomplished using the electron beam and the focused ion beam images in a dual beam system. c) SEM images of CNT column sidewalls grown with different CVD processes. Magnification increases from top to bottom. observed real time. Beyond a certain applied strain value (typically $1-3 \%$ ), the CNTs within the column start buckling in a collective fashion starting from the bottom, and consecutive buckles keep forming until the compression stops. The initiation of the buckle formation from the bottom can be attributed to the fact that catalyst particles diffuse into the substrate, possibly causing the particles to shrink, and as the effective catalyst size gets smaller, the CNTs they are producing become smaller diameter during the course of the growth. ${ }^{[41]}$

Successive buckle formation is depicted in Figure 2a, showing an experiment where a CNT column was compressed to different strain values. This buckle formation is very regular and repeatable, provided that the probe and the CNT column are parallel and the lateral stiffness of the probe is large. Also, the number of buckles is proportional to the imposed deformation on the column. Upon the release of the force, the CNT columns exhibit large plastic deformation and a short elastic recovery, typically between $10-50 \mu \mathrm{m}$ $(\sim 2-10 \%$ of the original height), depending on the number of buckles formed..$^{[5]}$ Thus, the CNT structure can be utilized as a spring, by reversible compression of the buckled portion. In-situ SEM compression tests also reveal that during the elastic recovery and small deflection spring operation, the previously formed buckles "breathe". In other words, their wavelength becomes shorter as they get compressed, and longer as the structure is released. Further details of this deformation mechanism will be discussed in a separate paper. ${ }^{[38]}$

A typical force versus deformation curve of a CNT column made by the hydrogen anneal recipe is shown in Figure 2b. In this experiment, a circular CNT column with

Table 1. Comparison of measured structural properties of CNT forests grown by the three recipes in the present study.

\begin{tabular}{lccc}
\hline & Fixed catalyst argon anneal & Fixed catalyst hydrogen anneal & Floating catalyst \\
\hline Structural Density $\left(\mathrm{kg} / \mathrm{m}^{3}\right)$ & 8 & 15 & 200 \\
Volume Fraction of CNTs & $0.36 \%$ & $0.68 \%$ & $9.09 \%$ \\
Structural Porosity & $99.64 \%$ & $99.32 \%$ & $90.91 \%$ \\
Average CNT Outer Diameter $(\mathrm{nm})$ & 25.8 & 8.1 & 89.9 \\
Average CNT Inner Diameter $(\mathrm{nm})$ & 11.1 & 4.3 & 15.1 \\
Average Number of Walls & 21.7 & 5.6 & 110 \\
Average CNT-CNT Spacing $(\mathrm{nm})$ & 342 & 74 & 260 \\
CNT Areal Density $\left(\mathrm{CNTs} / \mathrm{cm}^{2}\right)$ & $8.54 \times 10^{8}$ & $1.84 \times 10^{10}$ & $1.47 \times 10^{9}$ \\
\hline
\end{tabular}



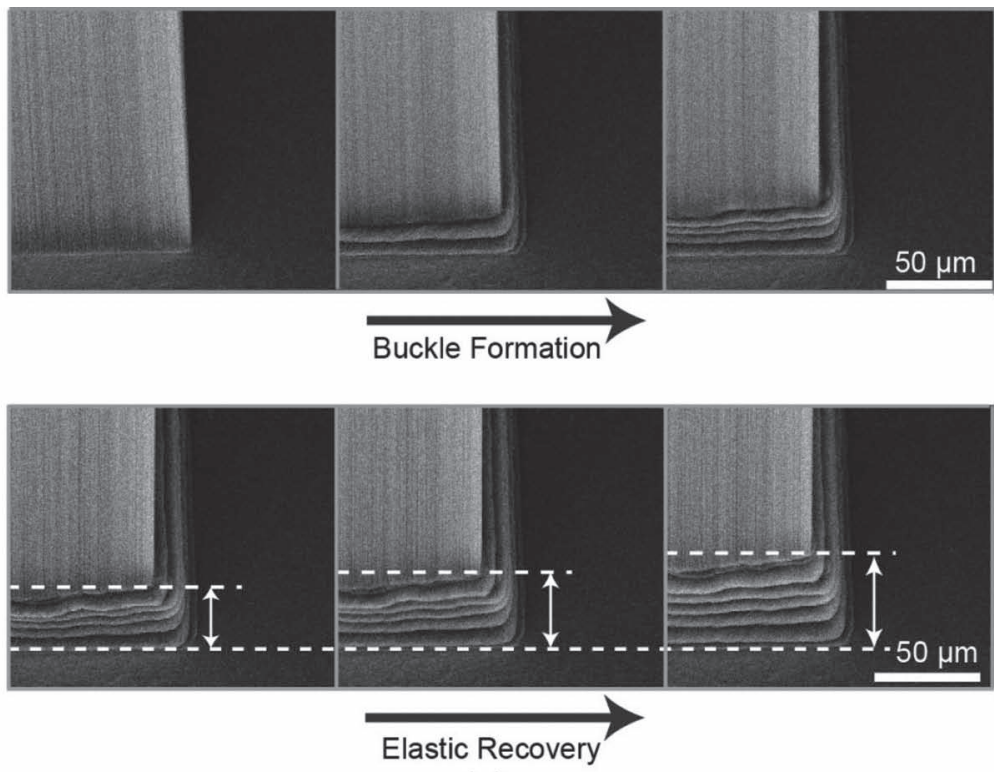

(a)

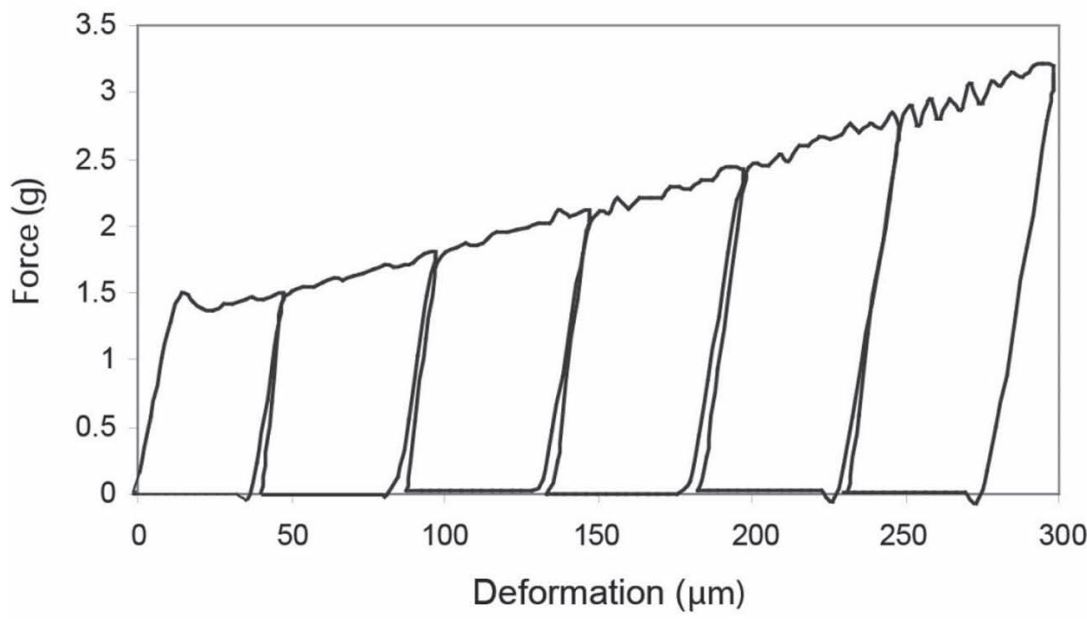

(b)

Figure 2. a) Compression of a fixed catalyst CNT column observed in-situ SEM: Successive buckle formation at the base of the column during compression and elastic recovery during release of the probe. b) Force-displacement plot upon successive loading and unloading of a CNT column grown by fixed catalyst method using hydrogen anneal.

a diameter of $400 \mu \mathrm{m}$ is compressed by $50 \mu \mathrm{m}$ increments to a total displacement of $300 \mu \mathrm{m}$, using a tribometer. First, the punch is brought down until it comes into contact with the CNT column. Alignment of the punch with the column is done by observing live images provided by two perpendicular cameras. This position is marked as $\mathrm{z}=0$ and during the remainder of the test, the deformations are defined with respect to this starting point. The column is first compressed by a pre-determined amount, typically $50 \mu \mathrm{m}$, and then released where the punch is brought back to $\mathrm{z}=0$. This compression routine is repeated by increasing the compression amount incrementally by $50 \mu \mathrm{m}$ each cycle. The first cycle starts with a linear force displacement relationship. After a certain force is reached, the column starts forming successive local buckles at the base.
During the release of the punch, the column recovers some of the compressed length elastically, but this is a small fraction of the overall deformation, and as shown in Figure 2a, none of the buckles recover fully. The second cycle starts with an elastic compression, with the same stiffness as observed during the elastic recovery of the first cycle. This is followed by successive buckle formation and again an elastic release. The test is continued until the desired number of incremental compressions is reached, and the incremental recovery behavior repeats. Another important observation is that within the elastic region, the stiffness of the CNT column is related to the number of buckles formed, i.e., the compliance of the column is proportional to the number of buckles. ${ }^{[5,38]}$

Figure 3a compares the resulting buckles on a column grown with fixed catalyst CVD with argon anneal to buckles on a column grown by fixed catalyst CVD with hydrogen anneal. Even though the argon anneal and hydrogen anneal forests exhibit the same fundamental mechanical behavior, i.e., successive buckle formation with large plastic deformation upon compression, for the case of argon anneal, the CNT columns are more compliant, and the localized plastic buckling mechanism is more pronounced. Also, for hydrogen anneal, the force fluctuation during the formation of the buckles is less pronounced in the force vs. deformation curve, and the average wavelength (vertical distance) of the buckles is shorter $(\sim 3.2 \mu \mathrm{m}$ vs. $\sim 4.5 \mu \mathrm{m})$.

To further investigate the difference between the two fixed catalyst methods, stiffness values versus cross-sectional area are compared in Figure 3b. As discussed earlier, each compression amount results in different number of buckles and hence in a different stiffness value, as measured during unloading and subsequent compression within the elastic range. Thus, stiffness values of columns with different cross-sectional areas are compared at the same strain (200 $\mu \mathrm{m}$ compression). The stiffness values of CNT columns grown with hydrogen anneal are four times larger than the stiffness of CNT columns grown with argon anneal. As the area increase translates into an increase in the number of CNTs in the column, the density increase should also translate directly into a proportional stiffness change, and this significant stiffness change can be attributed to the larger CNT packing density (20X) resulting from the hydrogen anneal method. However, the difference in CNT diameter also plays a role (hydrogen anneal results in $\sim 1 / 3^{\text {rd }}$ of the average CNT diameter compared to argon anneal, as listed in Table 1), and the alignment and bundling of the CNTs may be affecting the stiffness as well. As will be discussed later, this relationship is 

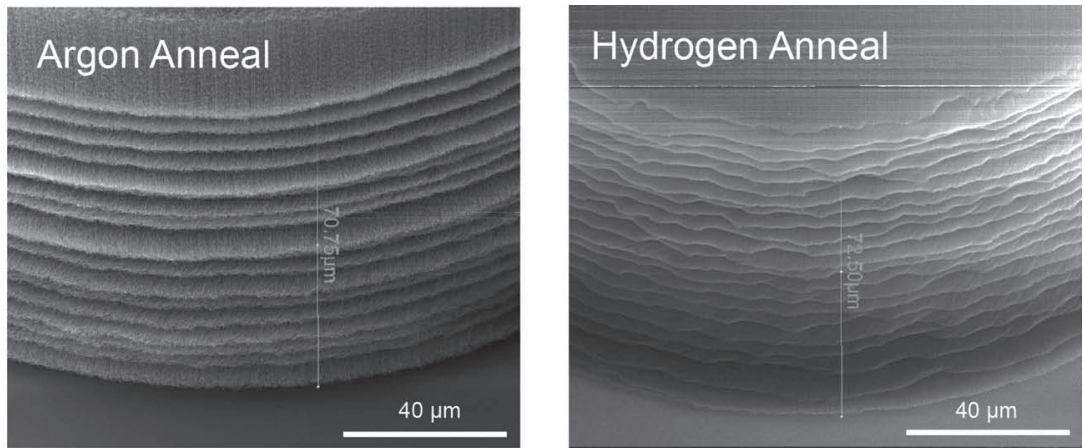

(a)

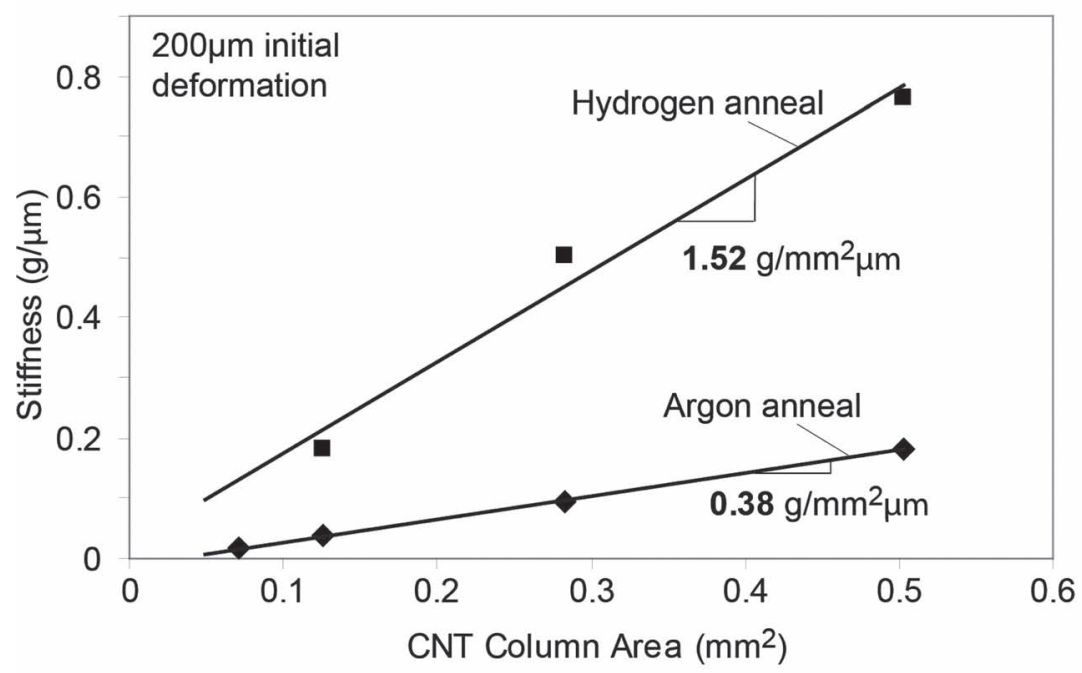

(b)

Figure 3. Comparison of deformation behavior and stiffness of CNT columns grown using argon anneal and hydrogen anneal fixed catalyst CVD recipes: a) Bottom sections of circular columns grown by fixed catalyst CVD after compressive loading and unloading; b) Relationship between stiffness and CNT column cross-sectional area. The stiffnes is proportional to the cross-sectional area and is four times higher for the hydrogen anneal recipe. The stiffness values correspond to the slope of the unloading curve after a $200 \mu \mathrm{m}$ initial compression. ${ }^{[5,38]}$

captured accurately by an open-cell foam model. This result demonstrates for the first time that the stiffness of CNT forests grown with fixed catalyst method can be tuned significantly simply by the CVD growth recipe, which directly influences the morphology of the CNT structure. Figure $3 \mathrm{~b}$ also shows that the stiffness of a CNT column is directly proportional to the area, thus demonstrating that all the CNTs in the structure contribute to the load-bearing capacity, and that the morphology is uniform inside the CNT column.

\subsection{Deformation Mechanism of High-Density CNT Columns Grown by the Floating Catalyst CVD Method}

For the case of growth with the floating catalyst, buckle formation is also observed, but the buckles recover almost fully after the force is released. This elastic behavior is observed during in-situ SEM compression, shown in Figure 4a. Also, as in the case of fixed catalyst growth, buckle formation starts at the bottom. This is attributed to the fact that during the CNT growth, the effective diameter of the CNTs constantly increase ${ }^{[30]}$ due to pyrolysis in the growth system. Because this is a "bottom-up growth" process (i.e., CNTs grow from the bottom surface of the column), the CNTs at the bottom have the smallest diameters due to the fact that they spent the least amount of time in the furnace and accumulate less amorphous carbon on their sidewalls. As a result, the CNT columns are weakest at the bottom.

Because CNT columns grown by the floating catalyst method behave differently than the columns grown with fixed catalyst method and recover after deformation, a simpler force-displacement sequence is used. Test results for various area columns are shown in Figure 4b for $50 \mu \mathrm{m}$ compression. As the columns are compressed, the stiffness increases, resulting in nonlinear behavior. To compare stiffness values of different area columns, the average stiffness values are calculated using the straight line connecting the maximum force and deflection with the initial point. Figure $4 \mathrm{c}$ plots the average stiffness values versus the column area. Again, the stiffness of CNT columns is proportional to the area, indicating that all of the CNTs in the structure contribute proportionally to the stiffness. CNT columns grown with the floating catalyst method are approximately 75 times stiffer than CNT columns grown with the argon anneal fixed catalyst method, and approximately 18 times stiffer than CNT columns grown with hydrogen anneal fixed catalyst method. These values are based on the slopes in Figures 3b and 4c.

This fundamental difference in deformation behavior and elastic properties between the low density CNT forests (fixed catalyst method) and high density CNT forests (floating catalyst) can be attributed to the relative influence of elastic restoring forces compared to van der Waals forces among CNTs in close proximity. We presume that van der Waals forces are strong enough to prevent elastic recovery of buckles among smaller diameter CNTs made by the fixed catalyst method, whereas the large diameter CNTs in forests grown with floating catalyst method are able to generate enough restorative force to overcome the Van der Waals forces and elastically recover. We consider that the deformation of CNT column within the elastic range is based on the bending of individual CNTs within the column. As the moment of inertia of a circular cross-section material is proportional to the fourth power of the diameter, the bending stiffness of a CNT in the case of floating catalyst method (average diameter $=89.9 \mathrm{~nm}$ ) is $150 \mathrm{X}$ larger than the bending stiffness of a CNT in the case of fixed catalyst method with argon anneal (average diameter = $25.8 \mathrm{~nm}$ ). While a detailed model of this behavior is beyond the 

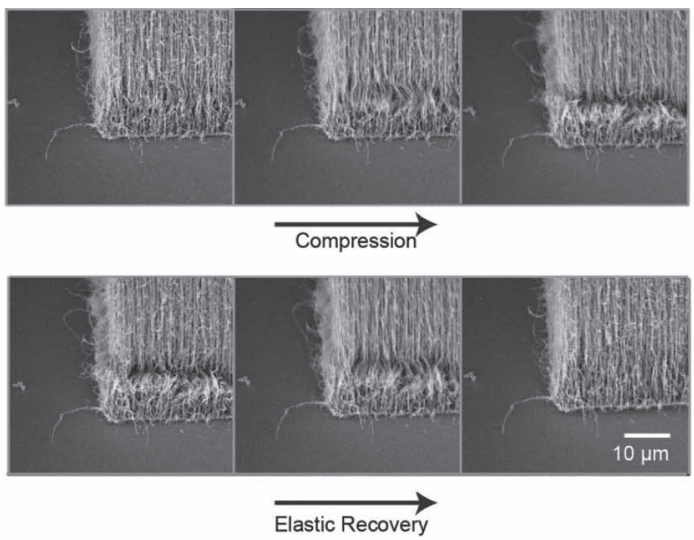

(a)

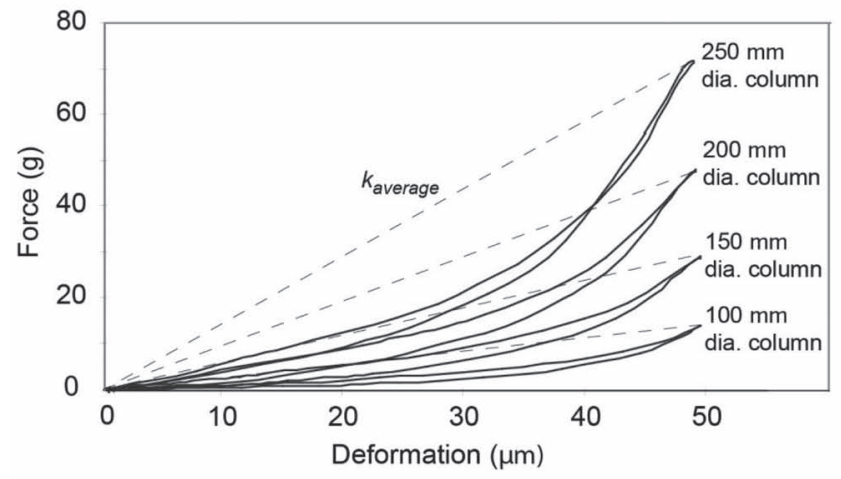

(b)

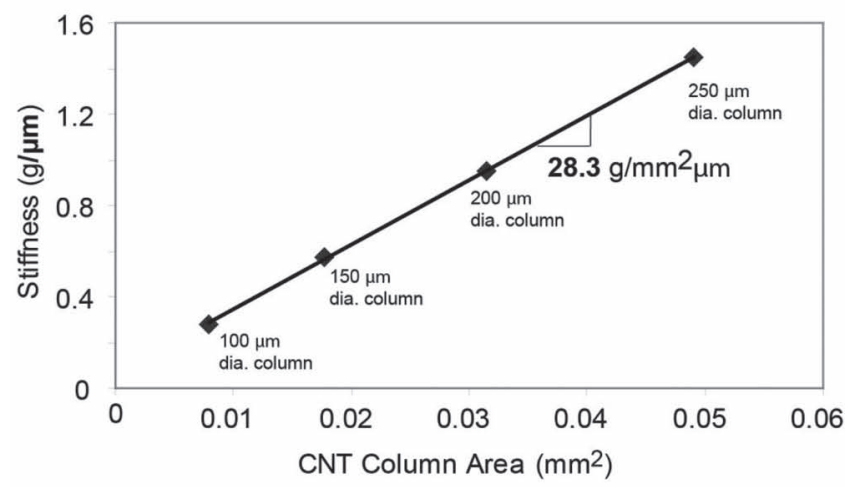

(c)

Figure 4. a) In-situ SEM compression close-up snapshots of the corner of a CNT column grown using floating catalyst recipe. b) Force-displacement relationships for different column diameters. c) Average stiffness versus column area, where the stiffness was calculated as indicated by the dashed line in (b).

scope of this study, the qualitative difference in elastic properties suggests why CNT forests comprising larger diameter CNTs exhibit elastic recovery after significant deformation.

\subsection{Ashby Chart and Comparison of CNT Growth Techniques}

The significant differences in stiffness and density resulting from the tested growth methods motivated us to compare the properties to conventional materials, using the modified Ashby chart ${ }^{[36]}$ shown in Figure 5. Although we compare the stiffness of CNT columns to conventional materials on the Ashby chart, we must note that these values are not the Young's Modulus. The Young's modulus is defined for structures and materials that exhibit a uniform strain field along the axis of loading, which is not the case for CNT structures, except possibly during the very short initial elastic region. Hence, these values are defined as "effective modulus". The effective modulus values for CNT columns are calculated as $E_{s}=k L / A$ where $L$ is the initial height of the column, $A$ is the cross-sectional area, and $k$ is the stiffness.

The Ashby chart shows that CNT forests span the density limits of conventional foam materials, and reach an order of magnitude higher stiffness at equivalent density. This may have important implications considering the other properties of CNTs, including electrical conductivity and thermal stability. It is interesting to note that as-grown CNT columns have a lower density than known aerogel materials for which elastic modulus has been reported. The lightest reported aerogel has a density of $4 \mathrm{~kg} / \mathrm{m}^{3} ;$; $[3]$ however, to our knowledge no elastic properties of this material have been reported. This implies that CNT forests can be useful in load-bearing materials in weight-sensitive applications. Also, since they can deform to very large strain levels plastically in the case of fixed catalyst CNTs, they could be suitable for applications where energy absorption is critical. For example a column grown with the fixed catalyst method could be completely deformed until all the aligned portion is consumed and turned into buckles, resulting in large energy absorption in the form of plastic deformation.

\subsection{Energy Storage Capacity}

The different growth methods also result in wide-range tuning of the mechanical energy storage capacity. Table 2 compares the effective modulus, strength and energy absorption capabilities of the CNT columns grown by each method. The energy absorption properties are calculated for elastic energy absorption, namely resilience, which is the property of a material to absorb energy elastically and release this energy upon unloading. In other words, it is the maximum energy per unit volume that can be elastically stored. This is represented by the area under the curve in the elastic region of the stress-strain curve:

$U_{r}=\frac{\sigma_{y}^{2}}{2 E}$

where $\sigma_{\gamma}$ is the yield strength and the $E$ is modulus of the material. This quantity is also called modulus of resilience. Using this definition, we find that CNT structures made by the floating catalyst method can store 3 orders of magnitude more elastic energy per unit volume compared to CNT structures grown with fixed catalyst method (argon anneal). If we look at the elastic energy stored per unit mass, the difference is more than 2 orders of magnitude. This demonstrates that the mechanical properties of CNT structures can be tuned for a wide range of applications. 


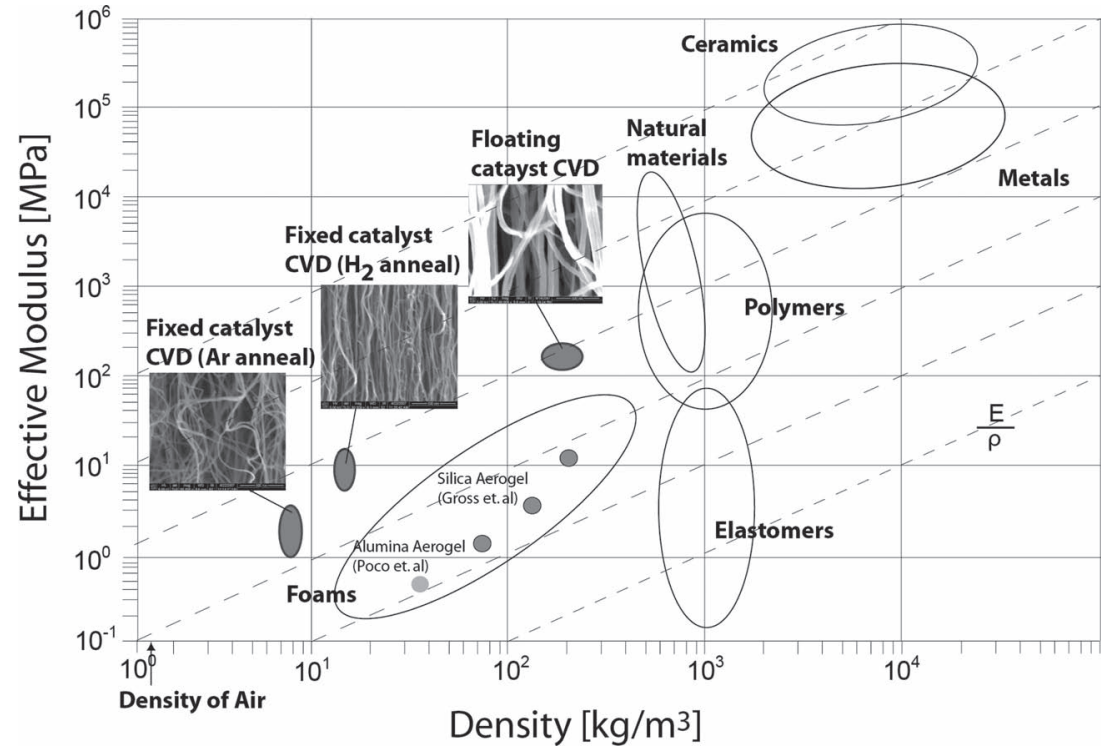

Figure 5. Ashby chart comparing mechanical properties of CNT columns grown in this study to conventional materials. The density values of the CNT columns span the range of conventional foamsbut they have approximately 10 -fold higher stiffness.

As mentioned earlier, one promising application of CNT columns is microsprings for electromechanical probing applications $^{[5]}$. Efficient materials for springs are the ones with largest modulus of resilience, or with the largest ratio of modulus of resilience to density. When we compare the ratio of the modulus of resilience to density for CNT columns and conventional engineering materials, we find that CNT forests grown with the floating catalyst method $[3.61 \mathrm{~kJ} / \mathrm{kg}]$ have comparable values to most engineering materials, such as Ti alloys $[0.2-2.6 \mathrm{~kJ} / \mathrm{kg}]$ and steel $[0.4-0.9 \mathrm{~kJ} / \mathrm{kg}]$, and others listed in Table 2. More importantly, the elastic energy storage properties of CNT forests can be tuned over a wide range as demonstrated in this study.

\subsection{Analogy with Open-Cell Foam Material}

The low density of CNT forests also warrants comparison of their deformation mechanisms to those of conventional foam materials. Due to the porous and entangled nature of the CNT forests, the deformation is dominated by the bending of individual CNTs within the array, which are held together by van der Waals forces. We suggest that CNT forests are analogous to open cell foam materials, which are also highly porous, and deform by bending of individual cell walls/edges. A visual analogy between CNT forests and open cell polyurethane is shown in Figure 6a-b.

Based on this analogy, we consider that the Young's modulus of a cellular structure can be approximated as

$$
E \sim \frac{t^{4}}{a^{4}}
$$

where $t$ is the thickness of the fibers and $a$ is the spacing between the fibers. ${ }^{[39]}$ In the case of CNT forests, the CNT diameter is analogous to the fiber thickness, and the average CNT-CNT spacing is analogous to the spacing between fibers. Using this relationship and the CNT diameter and spacing values listed in Table 1 , the structural stiffness ratio between the two fixed catalyst recipes, namely $\mathrm{E}_{\text {fixed(H2 anneal) }} / \mathrm{E}_{\text {fixed (Ar anneal), }}$, is predicted very well (4.43 compared to measured value of 4.02). On the other hand, the prediction for the modulus ratio between the floating and fixed catalyst recipes, namely $\mathrm{E}_{\text {(floating) }} / \mathrm{E}_{\text {fixed (Ar anneal) }}$ is not as close, (441 compared to measured value of 106.5). As discussed before, the deformation mechanism of the fixed and floating catalyst recipes are different and within the same type of deformation mechanism, namely fixed catalyst, the model predicts the modulus ratio very well.

A more accurate estimate for the case of floating catalyst may be obtained by considering only the load-bearing portion (effective length) of the CNT column. Additionally, the open-cell model predictions of the stiffness ratios between different CNT growth methods are very sensitive to the CNT diameter and CNT-CNT spacing. According to the model, the stiffness is proportional to the fourth power of the CNT diameter, and it is inversely proportional to the fourth power of the average CNT-CNT spacing. For example, if the measured average diameter for the case of fixed catalyst growth method with argon anneal was $30 \mathrm{~nm}$ instead of $25.8 \mathrm{~nm}$, and CNT-CNT spacing was $280 \mathrm{~nm}$ instead of $342 \mathrm{~nm}$, the modulus ratio between floating catalyst CNT columns and fixed catalyst (argon anneal) CNT columns would be 108, which is

Table 2. Comparison of measured mechanical properties of CNT forests grown by the different recipes.

\begin{tabular}{lccc}
\hline & Fixed Catalyst argon anneal & Fixed catalyst $H_{2}$ anneal & Floating catalyst \\
\hline Effective Modulus $\left.[\mathrm{MPa}]^{\mathrm{a}}\right)$ & $1.86(\text { Range: } 1.3-3.26)^{\mathrm{b}}$ & $7.44(\text { Range: } 5.2-13.04)^{\mathrm{b})}$ & 138.5 \\
Yield Strength $[\mathrm{MPa}]$ & 0.021 & 0.117 & 9.997 \\
Modulus of Resilience $\left[\mathrm{k} / \mathrm{m}^{3}\right]$ & 0.23 & 1.84 & $\left.721.65^{\mathrm{c}}\right)$ \\
Modulus of Resilience $/$ density ${ }^{\mathrm{d})}[\mathrm{kJ} / \mathrm{kg}]$ & 0.029 & 0.123 & $\left.3.608^{\mathrm{c}}\right)$ \\
\hline
\end{tabular}

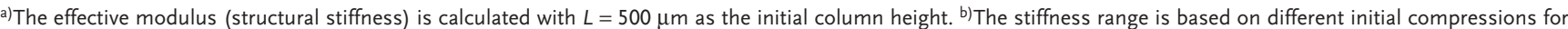
the case of fixed catalyst growth. ${ }^{c}$ The modulus of resilience values are calculated using Equation 1 with the assumption of a linear force/deformation curve. However, as discussed earlier, the elastic deformation for the floating catalyst is non-linear, which is ignored for the purposes of this discussion. d) Modulus of Resilience/density of common engineering materials: Ti alloys: 0.2-2.6, Steel: 0.4-0.9, CFRP (carbon fiber reinforced polymer): 3.9-6.5, wood: 0.3-0.7, Nylon: 1.3-2.1, rubber: 18-45. 


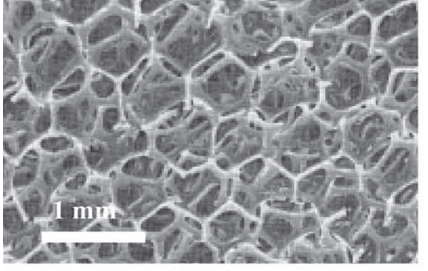

Open cell polyurethane

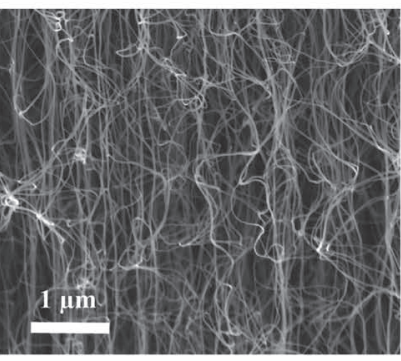

CNT Forest

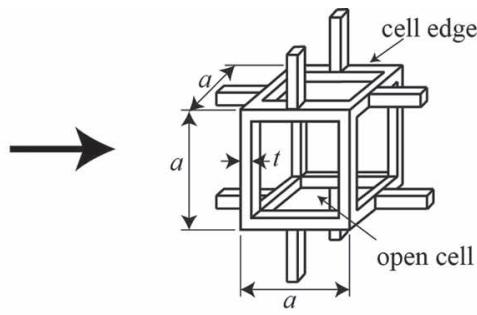

(a)

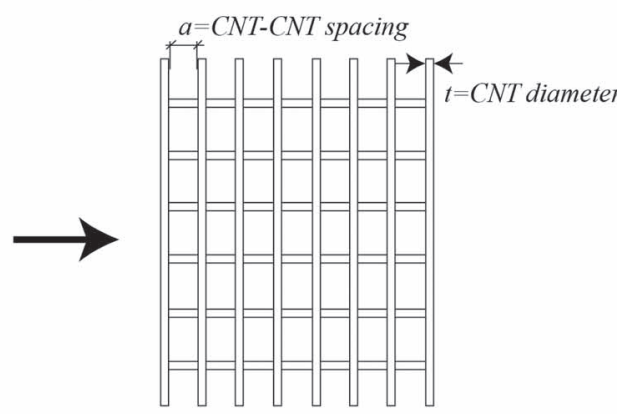

(b)

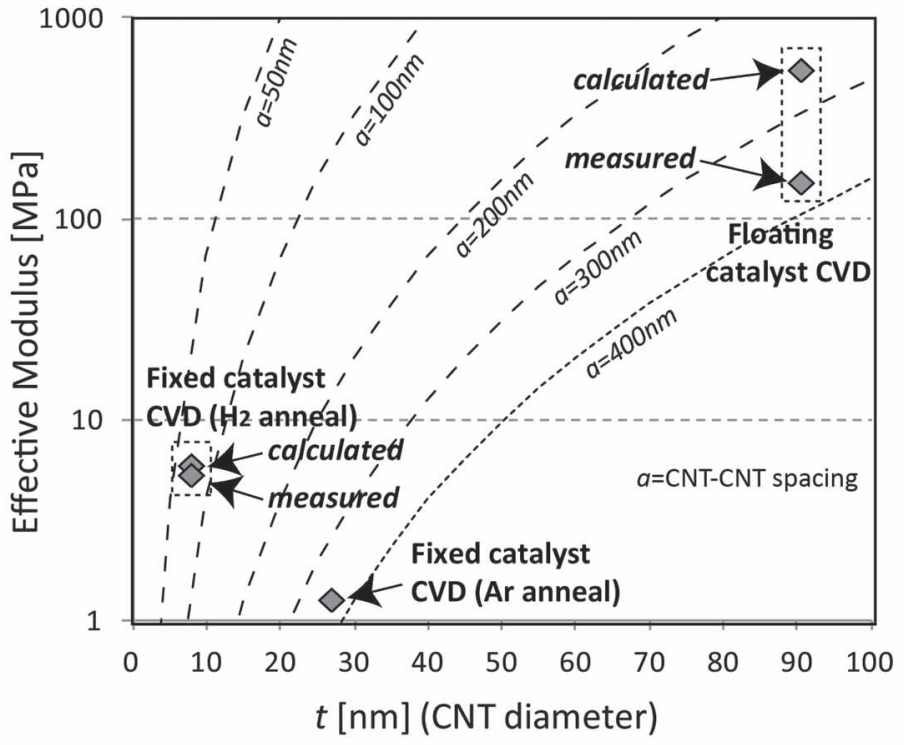

(c)

Figure 6. Analogy between open-cell foams and CNT forests. a) Microstructure of open-cell polyurethane foam and cubic model of an open-cell foam showing the edge length $I$ and the edge thickness $t$. Reproduced with permission. ${ }^{[39]}$ Copyright 1997, Cambridge University Press. b) SEM image of the sidewall of a CNT forest and schematic 2D open-cell model of a CNT forest. c) Effective modulus values for a range of CNT diameters and CNT-CNT spacing according to the open-cell model.

much closer to the measured ratio of 106. Another approximation in the model is that the cells are cubic, i.e., the CNTCNT spacing is the same in both lateral and vertical directions, which might be a substantial difference between the fixed and floating catalyst methods. The effective dimensions of the unit cell would depend on both the CNT density and alignment (waviness), and a more accurate measurement of the dimensions would be necessary to improve the accuracy of the model.
Despite these approximations, we now use the open-cell foam model to predict how the elastic properties can be tuned if it were possible to arbitrarily specify the CNT diameter and spacing. Figure 6e plots effective modulus values for a range of CNT diameters and CNT-CNT spacing according to the open cell model. The curves are based on the measured effective modulus for fixed catalyst growth with argon anneal. This plot also shows the measured values for the fixed catalyst growth method with hydrogen anneal and floating catalyst method, demonstrating that each method represents a limiting case of foamlike mechanical properties. As discussed earlier, the model predicts the effective modulus ratio between the two fixed catalyst growth methods accurately, and the measured value of the effective modulus with hydrogen anneal falls on the predicted value. For the floating catalyst case, both measured and calculated values are shown on the plot. Figure 6e shows, in a sense, the "design space" for CNT forests and the implications of the structural morphology on the elastic properties. It should be emphasized that these calculations show general dependencies and sensitivities of the elastic properties to the structural attributes. Although a further analysis considering the exact dimensions of the unit cells, and effects of CNT-CNT interactions is suitable for further study, this simple opencell model can be useful to establish design guidelines for the mechanical properties of CNT forests and selection of an appropriate synthesis method.

\section{Conclusion}

We showed that CNT forests can have a wide range of mechanical properties based on their growth recipe which determines the morphology of the forests, namely the CNT diameter and packing density. By comparing fixed and floating catalyst CVD growth methods, we showed that elastic moduli can span two orders of magnitude and energy absorption capacity can vary by 3 orders of magnitude. This paper not only demonstrated that within one growth method the stiffness of a CNT structure can be tuned by process parameters, but also that different recipes can result in CNT forests having a fundamentally different deformation mechanism and there is no universal deformation mechanism. High density and large-diameter CNT columns (floating catalyst) exhibit reversibility, whereas low denisity and smalldiameter CNT columns (fixed catalyst) exhibit large plastic deformation and can be pre-deformed to act as springs within a specified elastic range. We also showed that all of the CNTs in 
a CNT column contribute to the load-bearing capacity, and the stiffness of a CNT column is proportional to its cross-sectional area. We discussed the role of CNT diameter and packing density on the mechanical properties of CNT forests and proposed that the elastic behavior of CNT forests is analogous to opencell foams. We presented a simple model which can be useful to establish design guidelines for the mechanical properties of CNT forests and selection of appropriate synthesis method.

\section{Experimental Section}

CNT Growth and Patterned Column Fabrication: In the fixed catalyst growth method, CNTs were grown from a catalyst layer of 1.2/10 nm $\mathrm{Fe} / \mathrm{Al}_{2} \mathrm{O}_{3}$ film deposited by e-beam evaporation on a silicon wafer. The growth was performed in a 2 " quartz tube furnace (Lindberg) at atmospheric pressure. The furnace was ramped to the growth temperature of $750 \mathrm{C}$ under $400 \mathrm{sccm}$ Ar; then $400 / 150 \mathrm{sccm} \mathrm{H}_{2} / \mathrm{Ar}$ was introduced for 5 minutes, followed by $100 / 400 / 150 \mathrm{sccm} \mathrm{C}_{2} \mathrm{H}_{4} / \mathrm{H}_{2} / \mathrm{Ar}$ maintained for the growth period of 15 minutes. For the other variation of the fixed catalyst recipe studied here, hydrogen was supplied during the ramp time as well, instead of only the 5 minutes prior to growth. Also, the carrier gas used was helium instead of argon.

In the case of floating catalyst growth, the carbon source (xylene) and the catalyst precursor (Ferrocene- $\left.\mathrm{Fe}\left(\mathrm{C}_{5} \mathrm{H}_{5}\right)_{2}\right)$ were mixed (1\% ferrocene concentration) and pumped through a syringe pump $(6 \mathrm{ml} / \mathrm{h})$ into a heated steel bottle $(150 \mathrm{C})$ where they evaporate and are carried into the furnace $(750 \mathrm{C})$ with argon gas as the carrier gas $(300 \mathrm{sccm})$. More details about the growth systems and recipes can be found in Ref.[5]

The mechanical properties of the CNTs grown by each technique were characterized by compressing CNT columns of various cross-sectional areas. For the case of fixed catalyst method, shape control was achieved by patterning the catalyst layer from which CNTs grow. Similarly, for the case of floating catalyst growth, the area of the oxide layer was controlled by patterning a metal layer deposited earlier onto the oxide surface. For both growth techniques, patterning was achieved using a lift-off process with an image reversal photoresist. After the lithography step, the layer to be patterned $\left(\mathrm{Fe} / \mathrm{Al}_{2} \mathrm{O}_{3}\right.$ for fixed catalyst and $\mathrm{Au}$ for floating catalyst method) was deposited onto the photoresist and then lifted off in acetone. The masks used to pattern the Fe and Au layer have opposite polarities (shaded vs. non-shade areas) and the same geometric patterns. As an alternative to lithography, for both CVD techniques, laser machining of CNT arrays from full CNT films can also be used. ${ }^{[40]}$

For transmission electron microscopy (TEM), the CNT film was dispersed in an isopropanol bath and sonicated to separate the CNTs. Then the TEM grid was dipped into this solution with a tweezer and swept until some CNTs are captured onto the surface of the grid. The outer and inner diameters of CNTs were measured from the TEM images using microscopy software. The bulk densities of the CNT films were calculated by measuring the mass of a continuous CNT film with a sensitive balance and then dividing the mass by the volume of the CNT film. The volume fraction of the CNTs was calculated by dividing this value by the density of graphite $\left(2.2 \mathrm{~kg} / \mathrm{m}^{3}\right)$. The volume fraction of the CNTs can be also expressed as the area ratio of CNTs to the film area. For a unit area containing one CNT and average CNT-CNT spacing $a$, $V_{f}=A_{C N T} / A_{\text {total }}=\left(O D^{2}-I D^{2}\right) \pi /\left(4 a^{2}\right)$ where $V f$ is the volume fraction, $O D$ and $I D$ are average outer and inner diameters measured by TEM, and $a$ is average CNT-CNT spacing in the film. Using the measured values with TEM and the sensitive balance, average CNT-CNT spacing was calculated using the above equation.

Mechanical Testing: Mechanical characterization of CNT structures was performed ex situ with a CETR Micro Tribometer (www.cetr.com), as well as in situ SEM with a FEI dual beam system with an integrated OmniProbe (www.omniprobe.com), where a probe was inserted through a feed-through in the SEM chamber. The tribometer, which allows simultaneous measurement of applied force, displacement, and time, was used for measurements and more detailed testing and characterization. The in situ SEM probe was used to observe the CNT structures and record images during compression, and to gain insight into the deformation mechanism. For the tribometer measurements, the stiffness of the load sensor and the backlash of the leadscrew of the deformation stage were characterized and accounted for in the reported data. The CNT columns were compressed parallel to the CNT alignment direction. In all of the tests, the displacement was imposed at a constant rate of $10 \mu \mathrm{m} / \mathrm{s}$ and force was measured using a load sensor with a maximum range of $50 \mathrm{~g}(500 \mathrm{mN})$ and a resolution of $50 \mathrm{mg}(0.5 \mathrm{mN})$. For the in-situ SEM compression system, the perpendicularity of the probe to the sample holder was optimized to ensure mimum lateral compliance. Videos of the CNT structures under test were recorded during test and successive snapshot images were captured to analyze the deformation mechanism. More details about the measurement technique and test setup can be found in. ${ }^{[5]}$

\section{Supporting Information}

Supporting Information, including videos of the in-situ SEM compression tests, is available from the Wiley Online Library or from the author.

\section{Acknowledgements}

The authors gratefully acknowledge the support of FormFactor Inc. throughout the completion of this study. The authors would also like to thank Burag Yaglioglu for his help with TEM imaging, and John Gritters for fruitful discussions and support.

Received: March 27, 2012

Published online: July 26, 2012

[1] A. Cao, P. L. Dickrell, W. G. Sawyer, M. N. Ghasemi-Nejhad, P. M. Ajayan, Science 2005, 310, 1307-1310.

[2] Y. Gogotsi, Science 2010, 330, 1332-1333.

[3] A. Misra, J. R. Greer, C. Daraio, Adv. Mater. 2009, 21, 334-338.

[4] O. Yaglioglu, R. Martens, A. J. Hart, A. H. Slocum, Adv. Mater. 2008, 20, 357-362.

[5] O. Yaglioglu, Carbon Nanotube Based Electromechanical Probes, PhD Thesis, Massachusetts Institute of Technology, 2007.

[6] O. Yaglioglu, R. Martens, A. Cao, A. H. Slocum, in Proc. 57th IEEE Holm Conf. Electrical Contacts, Minneapolis, USA 2011.

[7] X. Li, G. Zhu, J. S. Dordick, P. M. Ajayan, Small. 2007, 3, 595599.

[8] M. S. Dresselhaus, Y. M. Lin, O. Rabin, A. Jorio, A. G. Souza, M. A. Pimenta, R. Saito, G. G. Samsonidze, G. Dresselhaus, Mater. Sci. Eng., C 2003, 23, 129-140.

[9] M. Dresselhaus, G. Dresselhaus, P. Avouris, Carbon nanotubes: Synthesis, Structure, Properties, and Applications, Springer, 2001.

[10] S. S. Fan, W. J. Liang, H. Y. Dang, N. Franklin, T. Tombler, M. Chapline, H. J. Dai, Physica E 2000, 8, 179-183.

[11] Z. W. Pan, S. S. Xie, B. H. Chang, L. F. Sun, W. Y. Zhou, G. Wang, Chem. Phys. Lett. 1999, 299, 97-102.

[12] Y. C. Choi, Y. M. Shin, Y. H. Lee, B. S. Lee, G. S. Park, W. B. Choi, N. S. Lee, J. M. Kim, App. Phys. Lett. 2000, 76, 2367-2369.

[13] G. Y. Zhang, D. Mann, L. Zhang, A. Javey, Y. M. Li, E. Yenilmez, Q. Wang, J. P. McVittie, Y. Nishi, J. Gibbons, H. J. Dai, Proc. Natl. Acad. Sci. USA 2005, 102, 16141-16145.

[14] L. B. Zhu, Y. H. Xiu, D. W. Hess, C. P. Wong, Nano Lett. 2005, 5, 2641-2645. 
[15] A. Cao, R. Baskaran, M. J. Frederick, K. Turner, P. M. Ajayan, G. Ramanath, Adv. Mater. 2003, 15, 1105.

[16] V. L. Pushparaj, L. Ci, S. Sreekala, A. Kumar, S. Kesapragada, D. Gall, O. Nalamasu, M. P. Ajayan, J. Suhr, Appl. Phys. Lett. 2007, 91, 153116

[17] L. J. Ci, Z. L. Rao, Z. P. Zhou, D. S. Tang, Y. Q. Yan, Y. X. Liang, D. F. Liu, H. J. Yuan, W. Y. Zhou, G. Wang, W. Liu, S. S. Xie, Chem. Phys. Lett. 2002, 359, 63-67.

[18] Y. Y. Fan, A. Kaufmann, A. Mukasyan, A. Varma, Carbon 2006, 44, 2160-2170.

[19] S. M. Huang, X. Y. Cai, C. S. Du, J. Liu, J. Phys. Chem. B. 2003, 107, $13251-13254$.

[20] X. S. Li, A. Y. Cao, Y. J. Jung, R. Vajtai, P. M. Ajayan, Nano Lett. 2005 , $5,1997-2000$

[21] W. C. Ren, F. Li, H. M. Cheng, J. Phys. Chem. B. 2006, 110, 16941-16946.

[22] G. B. Zheng, Y. F. Shi, J. W. Feng, J. K. Guo, J. Inorg. Mater. 2001, 16, 945-950.

[23] Z. P. Zhou, L. J. Ci, X. H. Chen, D. S. Tang, X. Q. Yan, D. F. Liu, Y. X. Liang, H. J. Yuan, W. Y. Zhou, G. Wang, S. S. Xie, Carbon 2003, 41, 337-342.

[24] R. Andrews, D. Jacques, A. M. Rao, F. Derbyshire, D. Qian, X. Fan, E. C. Dickey, J. Chen, Chem. Phys. Lett. 1999, 303, 467474.

[25] Q. Zhang, F. Du, L. Dai, J. Baur, Carbon 2011, 49, 386-397.

[26] C. McCarter, S. Mesarovic, D. Bahr, R. Richards, C. Richards, D. McClain, J. Jiao, J. Mater. Sci. 2006, 41, 7872-7878.

[27] S. Mesarovic, C. McCarter, D. Bahr, H. Radhakrishman, R. Richards, C. Richards, D. McClain, J. Jiao, Scripta Mater. 2007, $56,157-160$
[28] A. Qiu, D. F. Bahr, A. A. Zbib, A. Bellou, S. Dj. Mesarovic, D. McClain, W. Hudson, J. Jiao, D. Kiener, M. J. Cordill, Carbon 2011, 49, 1430-1438.

[29] S. B. Hutchens, L. J. Hall, J. R. Greer, Adv. Funct. Mater. 2010, 20 2338-2346.

[30] X. Li, Tailoring Vertically Aligned Carbon Nanotube Growth and Fabrication of Carbon Nanotube Membrane, PhD Thesis, Rensselaer Polytechnic Institute, 2007.

[31] X. Gui, J. Wei, K. Wang, A. Cao, H. Zhu, Y. Jia, Q. Shu, D. Wu, Adv. Mater. 2010, 22, 617-621.

[32] J. Suhr, P. Victor, L. Ci, S. Sreekala, X. Zhang, O. Nalamasu, P. M. Ajayan, Nat. Nanotechnol. 2007, 2, 417-421.

[33] J. R. Raney, A. Misra, C. Daraio, Carbon 2011, 49, 3631-3638.

[34] N. Yamamoto, E. J. Garcia, A. J. Hart, B. L. Wardle, A. H. Slocum, in Proc. of the 16th International Conference on Composite Materials, Kyoto, Japan 2007.

[35] G. D. Nessim, A. J. Hart, J. S. Kim, D. Acquaviva, J. Oh C. D. Morgan, M. Seita, J. S. Leib, C. V. Thompson, Nano Lett. 2008, 8, 3587-3593.

[36] M. F. Ashby, Materials Selection in Mechanical Design, ButterworthHeinemann Ltd, 2004

[37] R. W. Pekala, T. Tillostson, C. Alviso, J. Poco, J. LeMay, in Proc. MRS Symp., Boston, MA 1990

[38] O. Yaglioglu, R. Martens, A. Slocum, unpublished.

[39] L. J. Gibson, M. F. Ashby, Cellular Solids: Structure and Properties, Cambridge University Press, 1997.

[40] J. W. Elmer, O.Yaglioglu, R. D. Schaeffer, G. Kardos, O. Derkach, Carbon 2012, 509, 4114-4122.

[41] S.M Kim, C. L. Pint, P. B. Amama, D. N. Zakharov, R. H. Hauge, B. Maruyama, E. A. Stach, J. Phys. Chem. Lett. 2010, 1, 918-922. 